\title{
Isatuximab plus carfilzomib/dexamethasone versus carfilzomib/dexamethasone in patients with relapsed/refractory multiple myeloma: IKEMA Phase III study design
}

\author{
Philippe Moreau', Meletios A Dimopoulos², Kwee Yong ${ }^{3}$, Joseph Mikhael ${ }^{4}$, Marie-Laure \\ Risse $^{5}$, Gaëlle Asset ${ }^{6}$ \& Thomas Martin*,7 \\ ${ }^{1}$ Department of Hematology, University Hospital, Allée de I'lle Gloriette, Nantes, 44093, France \\ ${ }^{2}$ Department of Clinical Therapeutics, National \& Kapodistrian University of Athens, Stadiou 510562 Athens, Greece \\ ${ }^{3}$ Department of Hematology, University College London, London, UK \\ ${ }^{4}$ Department of Medicine, Translational Genomics Research Institute, City of Hope Cancer Center, Phoenix, AZ, AZ 85028, USA \\ ${ }^{5}$ Sanofi, Vitry-Alfortville, France \\ ${ }^{6}$ Sanofi, Chilly-Mazarin, France \\ ${ }^{7}$ Department of Hematology, University of California at San Francisco, San Francisco, CA, CA 94143, USA \\ *Author for correspondence: Tel.: +1 415353 2051; Tom.Martin@ucsf.edu
}

\begin{abstract}
Although the treatment of relapsed/refractory multiple myeloma has improved dramatically over the past decade, the disease remains incurable; therefore, additional therapies are needed. Novel combination therapies incorporating monoclonal antibodies have shown significant promise. Here we describe the design of a Phase III study (NCT03275285, IKEMA), which is evaluating isatuximab plus carfilzomib and low-dose dexamethasone, versus carfilzomib/dexamethasone in relapsed/refractory multiple myeloma. The primary end point is progression-free survival. Responses are being determined by an independent review committee using 2016 International Myeloma Working Group criteria, and safety will be assessed throughout. The first patient was recruited in November 2017, and the last patient was recruited in March 2019; 302 patients have been randomized, and the study is ongoing.
\end{abstract}

Clinical trial registration: NCT03275285

First draft submitted: 23 July 2019; Accepted for publication: 6 November 2019; Published online: 13 December 2019

Keywords: carfilzomib $\bullet$ CD38 • isatuximab • monoclonal antibody • Phase III trial • relapsed/refractory multiple myeloma • study protocol

Multiple myeloma (MM) is the third most common blood cancer, characterized by uncontrolled growth of bone marrow plasma cells leading to anemia, lytic bone lesions, overproduction of monoclonal immunoglobulins and frequent hypercalcemia. The abnormal production and accumulation of immunoglobulins can also lead to renal dysfunction and an increased risk of infection. Almost 160,000 new cases were diagnosed globally in 2016, with an associated estimate of 2.1 million disability-adjusted life-years [1]. At diagnosis, most patients present with advanced disease, with limited screening currently available to aid in early diagnosis.

A broad spectrum of therapeutic agents is currently available for treatment of patients with MM. These include immunomodulatory drugs (IMiDs) that increase the activation of $\mathrm{T}$ cells and natural killer (NK) cells, induce apoptosis and inhibit cell adhesion molecules [2], and proteasome inhibitors (PIs) that inhibit the catalytic site of the $26 \mathrm{~S}$ proteasome inducing apoptosis. Both of these classes of drugs, the IMiDs (including thalidomide, lenalidomide and pomalidomide) and the PIs (including bortezomib, carfilzomib and ixazomib), act through multiple mechanisms to cause myeloma cell death and prevent cell proliferation [3-9]. Although these drugs were initially used in relapsed myeloma, the use of IMIDs and PIs in induction therapies has resulted in better patient outcomes, including higher response rates and lower toxicity, when compared with traditional cytotoxic chemotherapy [3,10-12].

Future Medicine 
The initial treatment of MM is directed toward alleviating symptoms and decreasing the burden of disease [13]. A combination of an IMiD with a PI and a steroid (triplet therapy) has emerged as the mainstay of initial therapy [14]. Younger patients who are fit and have limited comorbidity may benefit from receiving consolidation therapy with autologous stem cell transplantation [15]. Following the induction and consolidation strategies, most patients receive maintenance therapy, with the goal of improving the duration of remission [16]. However, relapse is inevitable, and the vast majority of patients require additional therapy [17]. There are multiple salvage therapies available for patients who experience early relapse, but the ideal regimen or sequence of therapies has not been defined. Many factors, including patient-, disease- and treatment-related factors, are utilized to guide the selection of salvage therapy.

Several PIs are currently approved for use in relapsed refractory multiple myeloma (RRMM) only. Carfilzomib is a highly selective next-generation PI with minimal off-target adverse effects. In single-agent carfilzomib studies, the most frequently reported types of toxicity were cardiac events (e.g., congestive heart failure), pulmonary events (e.g., dyspnea) and renal impairment events (e.g., increased blood creatinine) [18]. This agent demonstrates superior efficacy to bortezomib with a more favorable adverse event (AE) profile, including lower rates of peripheral neuropathy. Carfilzomib is approved for treatment of RRMM as a single agent, as well as in combination with lenalidomide and/or dexamethasone $[19,20]$.

ENDEAVOR, a Phase III study conducted in 929 patients with RRMM after one to three prior treatments, showed superiority for carfilzomib plus dexamethasone versus the comparator bortezomib plus dexamethasone [21], which was utilized as a standard of treatment for RRMM for $>10$ years. These results led to the regulatory approval in 2016 of the carfilzomib plus dexamethasone combination in this patient population [21]. In addition, in the follow-up analysis of the overall survival (OS) benefit, carfilzomib provided a significant and clinically meaningful reduction in the risk of death, compared with bortezomib [21].

Also approved for the treatment of RRMM are a series of monoclonal antibodies that target cell membrane molecules that are highly expressed on the surface of MM cells [22-26]. Daratumumab targets CD38, a type II transmembrane glycoprotein that functions both as a signal-transducing receptor and a multifunctional ectoenzyme, and is highly expressed on MM cells. More than $90 \%$ of malignant plasma cells from patients with MM exhibit surface expression of CD38 [27], in contrast to its low-to-moderate expression on normal cells. Daratumumab was shown to have effects on CD38-expressing immunosuppressive Treg and B cells and myeloid-derived suppressor cells; the number, activation and clonality of cytotoxic $\mathrm{T}$ cells were increased after daratumumab treatment in heavily pretreated patients with RRMM [28]. Daratumumab is approved for use as a single agent in heavily pretreated patients with RRMM who are refractory to therapy with PIs and IMiDs [24] and for use in combination with pomalidomide/dexamethasone (USA only), lenalidomide/dexamethasone, bortezomib/dexamethasone and bortezomib/melphalan/prednisone as treatment for RRMM [29,30].

Elotuzumab targets SLAMF7, a glycoprotein that is selectively expressed on MM and NK cells. A Phase III study that compared elotuzumab plus lenalidomide/dexamethasone with lenalidomide/dexamethasone alone in patients with RRMM led to the approval of elotuzumab in combination with lenalidomide/dexamethasone in patients with MM who have received one to three prior therapies [22]. More recently, elotuzumab has been approved in combination with pomalidomide/dexamethasone in patients with RRMM who received at least two prior therapies, including lenalidomide and a PI [31].

\section{Isatuximab}

Isatuximab is an immunoglobulin G1 (IgG1) monoclonal antibody that targets a specific epitope on CD38. The mechanism of action of isatuximab is still being evaluated; however, it is known that isatuximab inhibits CD38 ectoenzyme activity and is capable of eliciting innate immune responses; antibody-dependent cellular cytotoxicity, complement-dependent cytotoxicity and antibody-dependent cellular phagocytosis, as well as the direct induction of target-cell killing through apoptotic mechanisms in the absence of cross-linking agents [23,32].

A recent preclinical study indicates that NK cell-mediated antibody-dependent cellular cytotoxicity is important [33]. In vivo, isatuximab likely also causes inhibition of immunosuppressive cells, including CD38 $\left.{ }^{+}\right)$Tregs and B regulatory cells, thereby producing additional antitumor effects (Figure 1) [34].

In preclinical studies, the antitumor effects of isatuximab appear to be significantly enhanced when it is combined with IMiDs and PIs. In a murine xenograft model utilizing MM.S1 cell lines, the combination of isatuximab plus carfilzomib showed there are additive antimyeloma effects [35]. In fact, while each individual agent slowed tumor growth, the combination demonstrated actual tumor regression [35]. It is unclear whether the mechanism for this synergy is through a proapoptotic effect or due to combined immune modulation. 


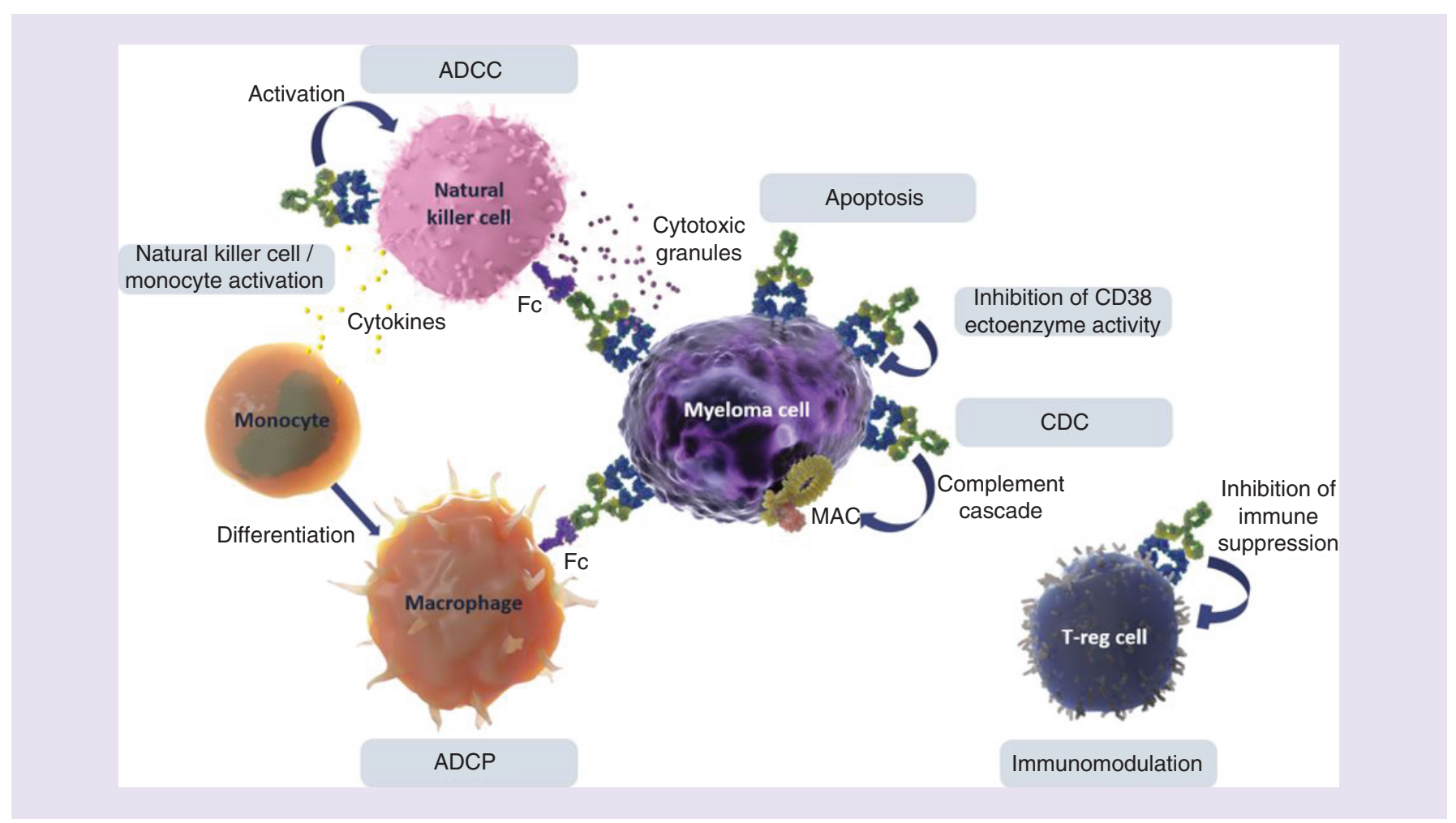

Figure 1. Isatuximab mechanism of action.

The clinical development program of isatuximab includes four ongoing Phase III clinical trials evaluating isatuximab in combination with currently available standard treatments for patients with RRMM or newly diagnosed with MM. Isatuximab has received orphan drug designation for RRMM by the US FDA. Isatuximab is also under investigation as an agent for the treatment of other hematologic malignancies (NCT03769181, NCT01084252) and solid tumors (NCT03367819, NCT03637764).

The first-in-human, Phase I/II study (NCT01084252) of isatuximab was carried out in advanced stage heavily pretreated patients with RRMM who had received a median of five prior lines of therapy. As a single agent, isatuximab demonstrated clinical activity [36,37] and was generally well tolerated in these patients. Infusion reactions (IRs) were the most common AEs that occurred, with most IRs being grade $1 / 2$ and generally observed during the initial infusion. The IRs were clinically manageable and resulted in few discontinuations, and no clinical sequelae were noted following recovery. At doses $\geq 10 \mathrm{mg} / \mathrm{kg}$, the overall response rate (ORR) was 20-29\%, the median time to first response was 4 weeks and the median duration of response was approximately 25 weeks [38]. Based on the clinical efficacy and favorable safety profile of isatuximab as a single agent, follow-up evaluation of isatuximab in combination with PIs and IMiDs is ongoing.

Isatuximab $10 \mathrm{mg} / \mathrm{kg}$ in combination with lenalidomide/dexamethasone (NCT01749969; Phase I) was also associated with an acceptable safety profile [39]. Overall, the safety profile of this combination was consistent with the safety profile of single-agent isatuximab and the combination of lenalidomide/dexamethasone alone $[39,40]$. Treatment of this heavily pretreated patient population (median five prior lines) with isatuximab plus lenalidomide/dexamethasone resulted in an ORR of 56\% [39]. The ORRs among patients who were refractory to lenalidomide or both an IMiD and a PI were $52 \%$ and $45 \%$, respectively. The ORR of the heavily pretreated population ( $\geq 3$ prior lines) was $48 \%(\mathrm{n}=44)$. The pharmacokinetics $(\mathrm{PK})$ of lenalidomide was not affected by co-administration with isatuximab [39]. In the Phase III ICARIA study evaluating the combination of isatuximab in combination with pomalidomide/dexamethasone, isatuximab plus pomalidomide/dexamethasone resulted in a median progression-free survival (PFS) of 11.5 months compared with 6.5 months in pomalidomide/dexamethasone alone, at median follow-up of 11.6 months $(\mathrm{p}=0.001)$. The ORR $(\geq \mathrm{PR})$ was $60.4 \%$ with isatuximab plus pomalidomide/dexamethasone compared with $35.3 \%$ with pomalidomide/dexamethasone alone ( $<0.0001)$ [41]. 
Table 1. Key eligibility criteria.

\begin{tabular}{|ll}
\hline Inclusion & Exclusion \\
\hline Aged $\geq 18$ years & Primary refractory multiple myeloma \\
\hline $\begin{array}{l}\text { Measurable disease: } \\
\text { serum M-protein } \geq 0.5 \mathrm{~g} / \mathrm{dl} \text { and/or urine M-protein } \geq 200 \mathrm{mg} / 24 \mathrm{~h}\end{array}$ & Patients with serum-free light chain measurable disease only \\
\hline Patients with RRMM with $1-3$ prior lines of therapy & Prior treatment with carfilzomib \\
\hline $\begin{array}{l}\text { Written informed consent } \\
\end{array}$ & ECOG performance status $>2$ \\
\hline $\begin{array}{l}\dagger \\
\text { Erogression on or within } 60 \text { days after the end of anti-CD38 therapy. }\end{array}$ & $\begin{array}{l}\text { Patients who are refractory to }{ }^{\dagger} \text { or failed to achieve at least a minimal response with } \\
\text { prior anti-CD38 therapy }\end{array}$ \\
\hline ECOG: Eastern Cooperative Oncology Group; MM: Multiple myeloma; RRMM: Relapsed/refractory MM. \\
\hline
\end{tabular}

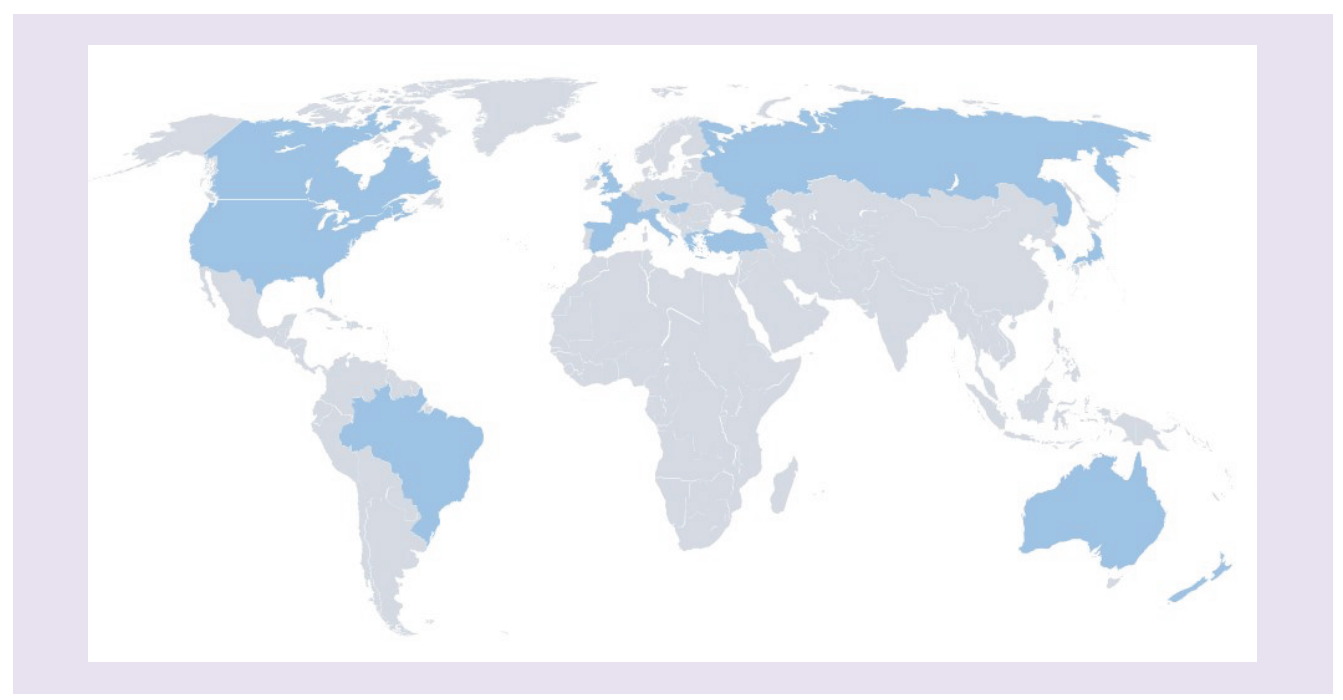

Figure 2. Recruiting countries. Darker shading indicates recruiting countries.

The combination of isatuximab with carfilzomib was investigated in a Phase Ib study (NCT02332850). Three dosing cohorts were evaluated: isatuximab $10 \mathrm{mg} / \mathrm{kg}$ every 2 weeks (Q2W), isatuximab $10 \mathrm{mg} / \mathrm{kg}$ weekly (QW) over 4 weeks then Q2W, and isatuximab $20 \mathrm{mg} / \mathrm{kg}$ weekly over 4 weeks then Q2W in combination with carfilzomib $\left(20 \mathrm{mg} / \mathrm{m}^{2}\right.$ on days 1 and 2 , and $27 \mathrm{mg} / \mathrm{m}^{2}$ on days $8,9,15$ and 16 in a standard 4-week cycle). As in previous isatuximab studies, dexamethasone was given routinely as a premedication for isatuximab. In 33 heavily pretreated patients with RRMM, all of whom had prior IMiD and PI exposure (79\% were double refractory), the ORR was $66 \%$ [42]. The combination was well tolerated, and the maximum-tolerated dose was not reached.

After applying the insights from the isatuximab clinical studies described above and the accompanying $\mathrm{PK} /$ pharmacodynamic analyses, the dosing schedule of isatuximab $10 \mathrm{mg} / \mathrm{kg}$ weekly for 4 weeks then Q2W was considered the optimal biological dose and was selected for further investigation in combination with other therapies, including carfilzomib, in the IKEMA Phase III study.

\section{IKEMA Phase III study}

Here we describe the design and rationale for an ongoing, prospective, multinational, randomized, open-label, parallel-group, two-arm, Phase III study (NCT03275285, IKEMA) evaluating the efficacy and safety of standard isatuximab $(10 \mathrm{mg} / \mathrm{kg} \mathrm{QW} \times 4$, then given Q2W) in combination with twice-weekly carfilzomib (3 weeks out of 4) and twice-weekly low-dose dexamethasone versus carfilzomib and low-dose dexamethasone alone in patients with RRMM. 
(A)
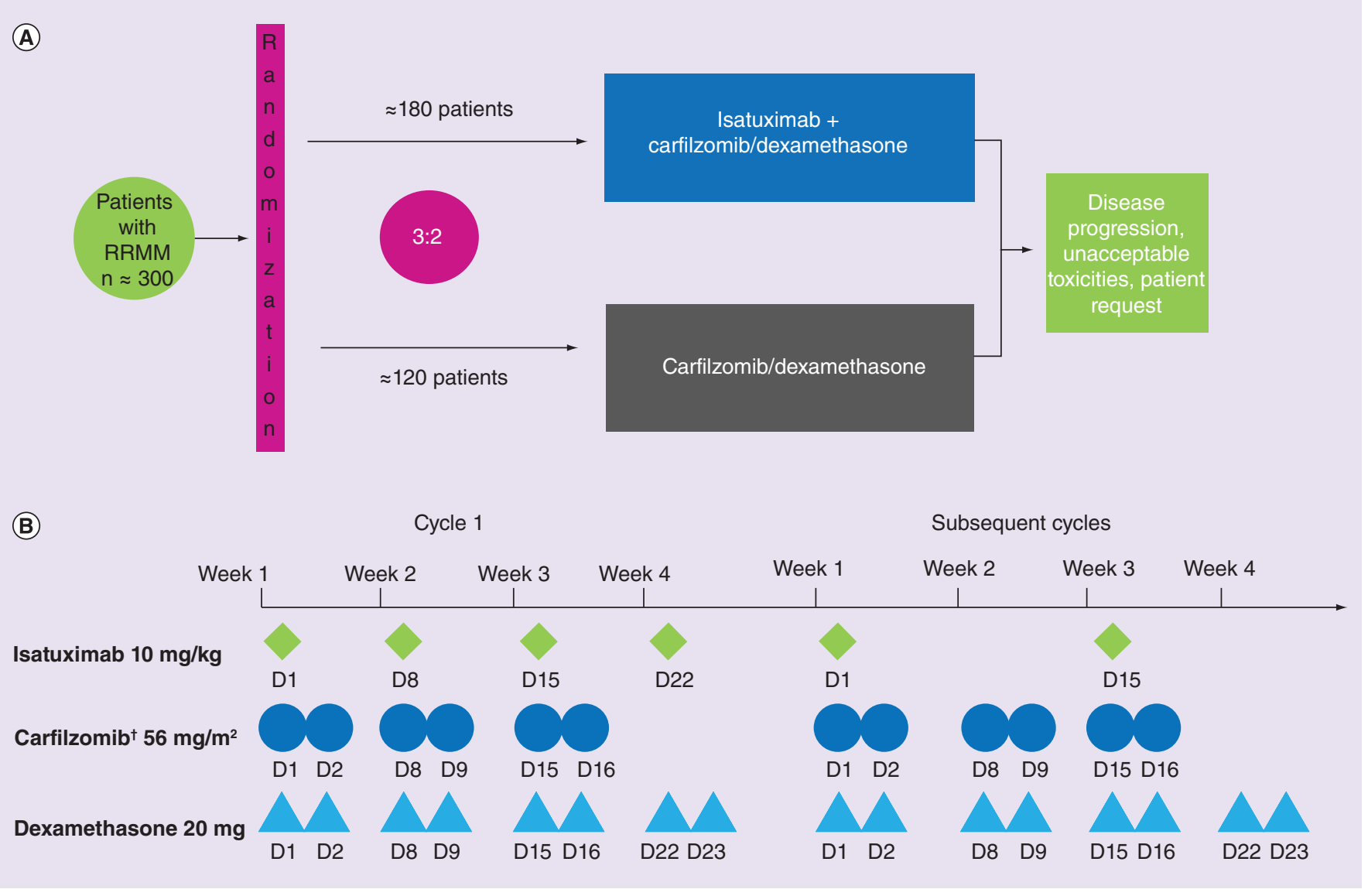

Figure 3. Study design and dosing schedule.

†Carfilozomib $20 \mathrm{mg} / \mathrm{m}^{2}$ day $1-2$, then $56 \mathrm{mg} / \mathrm{m}^{2}$ all additional days.

RRMM: Relapsed/refractory multiple myeloma.

\section{Objectives}

The primary objective of this study is to demonstrate the benefit of isatuximab plus carfilzomib/dexamethasone in prolonging PFS, compared with carfilzomib/dexamethasone alone, in the treatment of patients with RRMM. The key secondary objectives are to evaluate the ORR, the rate of very good partial response or better as per International Myeloma Working Group criteria [43], the rate of very good partial response or better with minimal residual disease negativity, the complete response rate and the OS of patients in each treatment arm. Additional secondary end points are being assessed, including safety, time to progression, PFS2 (the time to a second event of progressive disease or death), the duration of response, immunogenicity, PK and health-related quality of life in both arms.

\section{Key eligibility criteria}

Patients must be aged $\geq 18$ years with signed informed consent, with documented diagnosis of MM, and with measurable evidence of disease (serum M-protein $\geq 0.5 \mathrm{~g} / \mathrm{dl}$ and/or urine M-protein $\geq 200 \mathrm{mg} / 24 \mathrm{~h}$ ). Patients will have received at least one, but no more than three, prior lines of antimyeloma therapy, including IMiDs and PIs (Table 1).

Key exclusion criteria include Eastern Cooperative Oncology Group performance status greater than two; primary refractory MM (patients must have achieved at least a minimal response with any prior treatment during the disease course); serum-free light chain measurable disease only; refractoriness to prior anti-CD38 antibody therapy; prior carfilzomib therapy and/or prior allogeneic hematopoietic stem cell transplantation with active graft-versus-host disease (any grade or being under immunosuppressive treatment within the last 2 months); and a history of myocardial infarction, severe/unstable angina pectoris, coronary/peripheral artery bypass graft, New York Heart 
Table 2. Assessments and schedule.

\begin{tabular}{|c|c|}
\hline Timing & Assessment \\
\hline \multicolumn{2}{|l|}{ Patient characteristics and disease history } \\
\hline Baseline & $\begin{array}{l}\text { Demographics, myeloma history, and prior antimyeloma treatment, FISH (del[17p], } \\
t[4: 14], t[14: 16]) \text { to determine R-ISS stage }\end{array}$ \\
\hline \multicolumn{2}{|l|}{ Efficacy assessment ${ }^{\dagger}$} \\
\hline $\begin{array}{l}\text { Baseline, day } 1 \text { of all cycles, EOT }{ }^{\ddagger} \text {, follow-up (for patients who } \\
\text { discontinue the study treatment without PD) }\end{array}$ & $\begin{array}{l}\text { Serum M-protein, urine M-protein, serum-free light chains, quantitative } \\
\text { immunoglobulins }\end{array}$ \\
\hline Baseline, when indicated to document, overall response & Bone marrow disease involvement (plasma cell infiltration) \\
\hline Baseline and then annually & Bone lytic disease assessment \\
\hline Every 12 weeks (if present at baseline) & $\begin{array}{l}\text { Extramedullary disease assessment per investigator choice, with International } \\
\text { Myeloma Working Group response/failure criteria applied }\end{array}$ \\
\hline \multicolumn{2}{|l|}{ Safety } \\
\hline Continuously throughout the study period & $\begin{array}{l}\text { Adverse events, vital signs, physical examination, hematology, blood chemistry, } \\
\text { antidrug antibodies, electrocardiogram }\end{array}$ \\
\hline \multicolumn{2}{|l|}{ Isatuximab PK } \\
\hline $\begin{array}{l}\text { Days } 1,8,15 \text {, and } 22 \text { of cycle } 1 \text {, day } 1 \text { of subsequent cycles up to } 10 \\
\text { cycles }\end{array}$ & Isatuximab PK parameters by ELISA population PK modeling \\
\hline \multicolumn{2}{|l|}{ Patient-reported outcomes } \\
\hline Day 1 of all cycles, EOT $\ddagger, 90$ days after last administration & EORTC QLQ-C30, EORTC QLQ-MY20, EQ-5D-5L \\
\hline \multicolumn{2}{|c|}{$\begin{array}{l}\text { †Overall response and disease progression will be assessed by International Myeloma Working Group criteria (Kumar et al. 2016). } \\
\text { †EOT visit will be } 30 \text { days after last study treatment administration or before further antimyeloma therapy initiation, whichever comes first. } \\
\text { EORTC: European Organisation for Research and Treatment of Cancer; EOT: End of treatment; EQ-5D-5L: EuroQoL 5-dimensions questionnaire with } 5 \text { response levels } \\
\text { per dimension; PD: Progressive disease; PK: Pharmacokinetics; QLQ-C30: Quality-of-life questionnaire core module; QLQ-MY20: Quality-of-life questionnaire myeloma } \\
\text { module; R-ISS: Revised International Staging Score. }\end{array}$} \\
\hline
\end{tabular}

Association class III or IV congestive heart failure, greater than or equal to grade 3 arrhythmias, stroke/transient ischemic attack within last 6 months, and/or left ventricular ejection fraction lower than $40 \%$.

\section{Study design}

Study locations

This Phase III study has recruited patients with MM from Australia, Brazil, Canada, Czech Republic, France, Greece, Hungary, Italy, Japan, New Zealand, Spain, Republic of Korea, Russia, Turkey, the UK and the USA (Figure 2).

\section{Study design \& methodology}

Eligible patients have been randomly assigned, in a 3:2 ratio, to receive either isatuximab in combination with carfilzomib/dexamethasone or carfilzomib/dexamethasone alone. Treatment is given in 28-day cycles as follows: isatuximab $10 \mathrm{mg} / \mathrm{kg}$ intravenously (iv.) on cycle 1 at days $1,8,15$ and 22 then days 1 and 15 in subsequent cycles; carfilzomib iv. on cycle 1 at days 1 and 2 at $20 \mathrm{mg} / \mathrm{m}^{2}$ over $30 \mathrm{~min}$ then on cycle 1 at days $8,9,15$, and 16 , and on all subsequent doses at $56 \mathrm{mg} / \mathrm{m}^{2}$ over $30 \mathrm{~min}$ and also dexamethasone $20 \mathrm{mg}$ on days $1,2,8,9,15,16,22$, and 23 of every cycle; or carfilzomib/dexamethasone alone with the same schedule and dose (Figure 3).

Randomization will be stratified by a number of prior lines of therapy ( $1 \mathrm{vs}>1)$ and disease stage as determined by the Revised International Staging Score (R-ISS; I or II vs III vs not classified). Treatment will be continued until disease progression, unacceptable AEs or patient request.

Premedication will be included to reduce the severity of IRs with isatuximab and carfilzomib. Premedication for isatuximab will include diphenhydramine $25-50 \mathrm{mg}$ iv., ranitidine $50 \mathrm{mg}$ iv. and acetaminophen 650-1000 mg or equivalent. Dexamethasone will be given prior to isatuximab infusions in the isatuximab plus carfilzomib/dexamethasone arm and prior to carfilzomib infusion in the carfilzomib/dexamethasone arm as part of the premedication and as part of the treatment throughout the study. No postinfusion prophylaxis with dexamethasone will be required.

\section{Outcome measurements/end points}

Table 2 describes the assessment schedules for all measures. Demographic characteristics, including medical/surgical history, prior MM history (time from diagnosis, stage as defined by ISS at diagnosis, and prior anti-MM therapy), 
and the responses to prior treatments, will be collected at baseline. Disease characteristics, including M-protein subtype, extent of bone marrow and extra-medullary disease, cytogenetics (assessed by a central laboratory) and R-ISS, will also be recorded at baseline.

The 2016 International Myeloma Working Group criteria [43] will be applied by an independent response review committee to evaluate response and disease progression. Assessments will be made on the first day of every cycle and when treatment is stopped. PFS - defined as the time from randomization to either the first documented occurrence of progressive disease or the death of the patient from any cause, whichever occurs earliest - is the primary efficacy end point. Response will be assessed during follow-up and until disease progression in patients who discontinue therapy prior to disease progression. Subgroup analyses of PFS (e.g., by cytogenetic risk status, number of prior lines of treatment) will also be conducted. Next-generation sequencing will be used to assess minimal residual disease.

Safety evaluations will include vital signs, hematology and biochemistry assessments, electrocardiograms, and AEs: these will be followed throughout the study. AEs will be graded according to the National Cancer Institute Common Terminology Criteria for AEs v4.03. Immunogenicity will be assessed during study treatment. An indirect Coombs test will be performed at baseline and after treatment initiation in the isatuximab plus carfilzomib/dexamethasone arm only.

Patient-reported outcome assessments will be measured on day 1 of every cycle, at the end of treatment and 90 days after study treatment administration by using the patient-reported outcome/health-related quality of life and health utility instruments (European Organization for Research and Treatment of Cancer quality-of-life questionnaires C30 and MY20 [EORTC QLQ-C30 and QLQMY20] and the EuroQoL questionnaire EQ-5D-5L).

\section{Statistical considerations}

Analysis set

All patients who provided informed consent and were randomized will be included in the intent-to-treat (ITT) population, irrespective of whether they received any study treatment or not. Efficacy parameters will be analyzed primarily in the ITT population. The treatment arm assigned at randomization will be used in the ITT analyses.

The safety analysis population will comprise all members of the ITT population who are treated with at least one full or partial dose of study medication. Safety population analyses will be performed on the basis of the treatment received.

Quality of life will be assessed for patients from the safety population who complete the baseline visit and have at least one postbaseline assessment.

\section{Statistical analysis}

Analysis of the primary end point will be performed on the ITT population and consists of PFS comparison using a log-rank test procedure, stratified by stratification factors (number of prior lines and R-ISS stage) at the time of randomization. Safety analyses will be summarized utilizing descriptive statistics.

\section{Sample size}

A total of 302 patients have been randomized in a 3:2 ratio to receive isatuximab plus carfilzomib/dexamethasone and carfilzomib/dexamethasone, respectively.

\section{Discussion}

The treatment of MM has changed significantly over the past 10 years, with multiple new drug approvals and many novel combinations resulting in improved life expectancy. However, the majority of patients continue to have relapses after therapy, so additional novel agents and combinations for initial therapy and treatment of relapsed/refractory disease are needed in order to improve both survival and quality of life of patients with MM.

The pivotal Phase III IKEMA study is assessing potential improvement in PFS in patients receiving isatuximab. Preclinical studies demonstrated at least additive effects from the combination with isatuximab and carfilzomib, possibly by enhancing apoptosis. In addition, the combination of isatuximab with carfilzomib was investigated in a Phase Ib study in which encouraging efficacy and tolerability were observed [42].

Previous studies have examined PFS and other measures of efficacy in this RRMM population. Improved results were seen predominantly with triplet-based combinations, albeit with an increased risk of AEs [44]. The anti-CD38 antibody daratumumab was evaluated in two large Phase III trials: one compared daratumumab in combination with bortezomib/dexamethasone (DVd) versus bortezomib/dexamethasone (Vd) [45]; the second compared dara- 
Table 3. Studies of isatuximab for the treatment of multiple myeloma.

\begin{tabular}{|c|c|c|c|}
\hline NCT number & Combination & Status & Patient population \\
\hline \multicolumn{4}{|l|}{ Phase I or Phase I/II } \\
\hline NCT01749969 Phase I/II & ISA, Len, dex & Active not recruiting & RRMM, $\geq 2$ prior lines of therapy including IMiD \\
\hline NCT01084252 Phase I/II & ISA, dex & Active not recruiting & $\begin{array}{l}\text { Refractory } \mathrm{MM}, \geq 3 \text { prior lines of therapy including IMiD (for } \geq 2 \\
\text { cycles or } \geq 2 \text { months) and } \mathrm{PI} \text { (for } \geq 2 \text { cycles or } \geq 2 \text { months) }\end{array}$ \\
\hline NCT02332850 Phase lb & ISA, Car & Recruiting & $\begin{array}{l}\text { RRMM, } \geq 2 \text { prior lines of therapy including IMiD (for } \geq 2 \text { cycles or } \\
\geq 2 \text { months) and } \mathrm{PI} \text { (for } \geq 2 \text { cycles or } \geq 2 \text { months) }\end{array}$ \\
\hline NCT02514668 Phase I & ISA & Active not recruiting & $\begin{array}{l}\text { RRMM, } \geq 3 \text { prior lines of therapy including IMiD (for } \geq 2 \text { cycles or } \\
\geq 2 \text { months) and } \mathrm{PI} \text { (for } \geq 2 \text { cycles or } \geq 2 \text { months) }\end{array}$ \\
\hline $\begin{array}{l}\text { NCT02513186 } \\
\text { Phase I }\end{array}$ & $\begin{array}{l}\text { ISA }+ \text { VCD or ISA + } \\
\text { VRD }\end{array}$ & Recruiting & Newly diagnosed MM \\
\hline $\begin{array}{l}\text { NCT02812706 } \\
\text { Phase I/II }\end{array}$ & ISA & Active not recruiting & RRMM, $\geq 3$ prior lines of therapies including IMiD and PI \\
\hline $\begin{array}{l}\text { NCT03194867 } \\
\text { Phase I/II }\end{array}$ & ISA, Cem & Active not recruiting & $\begin{array}{l}\text { RRMM, } \geq 3 \text { prior lines of therapy including IMiD (for } \geq 2 \text { cycles or } \\
\geq 2 \text { months) and } \mathrm{PI} \text { (for } \geq 2 \text { cycles or } \geq 2 \text { months) }\end{array}$ \\
\hline $\begin{array}{l}\text { NCT03733717 } \\
\text { Phase I }\end{array}$ & ISA & Active not recruiting & $\begin{array}{l}\text { Refractory } \mathrm{MM}, \geq 2 \text { prior lines of therapy including IMiD (for } \geq 2 \\
\text { cycles or } \geq 2 \text { months) or } \mathrm{PI} \text { (for } \geq 2 \text { cycles or } \geq 2 \text { months) }\end{array}$ \\
\hline $\begin{array}{l}\text { NCT02283775 } \\
\text { Phase I }\end{array}$ & ISA, Pom, dex & Active not recruiting & RRMM, $\geq 2$ prior lines of therapy including Len and PI \\
\hline \multicolumn{4}{|l|}{ Phase II } \\
\hline $\begin{array}{l}\text { NCT02960555 } \\
\text { Phase II }\end{array}$ & ISA & Active not recruiting & Smoldering $\mathrm{MM}$ \\
\hline $\begin{array}{l}\text { NCT03104842 } \\
\text { Phase II }\end{array}$ & ISA, Car, Len, dex & Recruiting & Newly diagnosed MM \\
\hline \multicolumn{4}{|l|}{ Phase III } \\
\hline $\begin{array}{l}\text { NCT03275285 } \\
\text { Phase III }\end{array}$ & $\begin{array}{l}\text { ISA, Car, dex } \\
\text { or } \\
\text { Car, dex }\end{array}$ & Active not recruiting & RRMM, 1-3 prior lines of therapy \\
\hline $\begin{array}{l}\text { NCT03319667 } \\
\text { Phase III }\end{array}$ & Isa + VRD or VRD & Active not recruiting & Newly diagnosed MM \\
\hline $\begin{array}{l}\text { NCT02990338 } \\
\text { Phase III }\end{array}$ & $\begin{array}{l}\text { ISA, Pom, dex } \\
\text { or } \\
\text { Pom, dex }\end{array}$ & Active not recruiting & $\begin{array}{l}\text { RRMM, } \geq 2 \text { prior lines of therapy including Len (for } \geq 2 \text { cycles) and } \\
\text { PI (for } \geq 2 \text { cycles) }\end{array}$ \\
\hline $\begin{array}{l}\text { NCT03617731 } \\
\text { Phase III }\end{array}$ & ISA + VRD or VRD & Recruiting & Newly diagnosed MM \\
\hline \multicolumn{4}{|c|}{$\begin{array}{l}\text { Information collected from clinicaltrials.gov, } 14 \text { June } 2019 . \\
\text { Car: Carfilzomib; Cem: Cemiplimab; dex: Dexamethasone; IMiD: Immunomodulatory agent; ISA: Isatuximab; Len: Lenalidomide; MM: Multiple myeloma; PI: Proteasome inhibitor } \\
\text { Pom: Pomalidomide; RRMM: Relapsed/refractory multiple myeloma; VCD: Bortezomib, cyclophosphamide, dexamethasone; VRD: Bortezomib, lenalidomide, dexamethasone. } \\
\text { Adapted with permission from [34]. }\end{array}$} \\
\hline
\end{tabular}

tumumab in combination with lenalidomide/dexamethasone (DRd) versus lenalidomide/dexamethasone (Rd) [46]. Median PFS for the DVd triplet was 17 months [47]; for the DRd triplet, the median PFS was 44.5 months [48]. Daratumumab is approved for use in combination with bortezomib/dexamethasone and lenalidomide/dexamethasone and, in the USA, with pomalidomide/dexamethasone. More recently, a Phase Ib trial of daratumumab with carfilzomib/dexamethasone (D-Kd) has also reported promising activity in an RRMM population in which 60\% of patients were refractory to lenalidomide. ORR was $84 \%$ with a median follow-up of 16.6 months, and median PFS was not reached [49]. A Phase III trial evaluating isatuximab plus pomalidomide/dexamethasone versus pomalidomide/dexamethasone was recently completed and will provide information regarding this combination $[34,41]$.

IKEMA is the first Phase III study to evaluate isatuximab in combination with carfilzomib $\left(20-56 \mathrm{mg} / \mathrm{m}^{2}\right)$ and dexamethasone. The control arm doublet carfilzomib/dexamethasone was previously demonstrated to be an effective therapeutic regimen and was the first treatment to show OS superiority in patients with RRMM [21]. These drugs each have potent single-agent activity and little overlapping toxicity, which has provided significant enthusiasm for this therapeutic combination.

Although daratumumab and isatuximab are both anti-CD38 monoclonal antibodies, they bind to different epitopes [23]. In vitro, isatuximab is the only anti-CD38 antibody that induces direct apoptosis without cross- 
linking [23,32]. Whether these in vitro differences will translate into differences in clinical activity or improved activity in specific combinations remains unknown.

The IKEMA protocol offers an IMiD-free treatment option that may be particularly suitable for patients with disease progression after an IMiD-containing first-line therapy or those who are refractory to IMiDs. As summarized in Table 3, additional ongoing studies are evaluating the use of isatuximab with the current standard-of-care regimens in patients with newly diagnosed MM, smoldering MM and RRMM.

\section{Executive summary}

\section{Multiple myeloma}

- Multiple myeloma is characterized by plasma cell proliferation in the bone marrow and is associated with the production of excessive monoclonal immunoglobulins. Although in recent years survival rates have improved, most patients will relapse. Treatment for relapsed/refractory multiple myeloma (RRMM) remains an unmet need.

Isatuximab

- Isatuximab is a monoclonal antibody that targets a specific epitope on CD38 that is different from the binding site for daratumumab. Isatuximab has shown antitumor activity as a single agent and enhanced activity when combined with either immunomodulatory drugs or proteasome inhibitors in Phase I/II studies of heavily pretreated patients with RRMM.

\section{IKEMA}

- Herein, we describe the design of the Phase III IKEMA study (NCT03275285), which is evaluating isatuximab in combination with carfilzomib and low-dose dexamethasone versus carfilzomib/dexamethasone alone in patients with RRMM.

Objectives

- The primary objective of the IKEMA study is to demonstrate the benefit of isatuximab in combination with carfilzomib/dexamethasone in the prolongation of progression-free survival (PFS) compared with carfilzomib/dexamethasone alone in patients with RRMM. The key secondary efficacy objectives include evaluation of the overall response rate, the rate of very good partial response or better (2016 International Myeloma Working Group criteria), the rate of very good partial response or better with minimal residual disease negativity, the complete remission rate and overall survival in both treatment arms in patients with RRMM.

Key eligibility

- Eligible patients for the IKEMA study are adults with RRMM and confirmed disease progression within 60 days of the last therapy who have received between one and three prior lines of therapy, including lenalidomide and a proteasome inhibitors, alone or in combination.

Study design

- A total of 302 patients have been randomized in a 3:2 ratio to receive isatuximab plus carfilzomib/dexamethasone or carfilzomib/dexamethasone alone in 28-day cycles. Patients will continue therapy until disease progression, unacceptable toxicity or a patient request to discontinue.

Statistical considerations

- The primary end point is PFS. The intent-to-treat population is the primary population for all efficacy parameters. Comparison of PFS between treatment arms will be performed using a log-rank test procedure. The safety population will include intent-to-treat patients who have received at least part of a dose of the study treatments. Safety evaluations will include adverse events, laboratory parameters, vital signs, electrocardiogram results and physical examinations.

\section{Financial \& competing interests disclosure}

P Moreau has received honoraria from Amgen, Takeda, Celgene, Janssen and Novartis; a consulting/advisory role with Amgen, Takeda, Celgene, Janssen and Novartis. MA Dimopoulos has a consulting/advisory role for Celgene, Onyx, Janssen and Novartis. K Yong has a consulting/advisory role with Amgen, Janssen and Takeda; is in Speaker's bureau for Amgen and Takeda; received research funding from Amgen and Sanofi. J Mikhael received research funding from AbbVie, Celgene, Onyx and Sanofi. M-L Risse and G Asset are the employees of Sanofi. T Martin received research funding from Sanofi, Amgen and Seattle Genetics. The authors have no other relevant affiliations or financial involvement with any organization or entity with a financial interest in or financial conflict with the subject matter or materials discussed in the manuscript apart from those disclosed.

Medical writing assistance is provided by Louise Wright of Adelphi Communications Ltd, funded by Sanofi.

Open access

This work is licensed under the Attribution-NonCommercial-NoDerivatives 4.0 Unported License. To view a copy of this license, visit http://creativecommons.org/licenses/by-nc-nd/4.0/ 


\section{References}

Papers of special note have been highlighted as: $\bullet \bullet$ of considerable interest

1. Cowan AJ, Allen C, Barac A et al. Global burden of multiple myeloma: a systematic analysis for the global burden of disease study 2016. JAMA Oncol. 4(9), 1221-1227 (2018).

2. Holstein SA, McCarthy PL. Immunomodulatory drugs in multiple myeloma: mechanisms of action and clinical experience. Drugs 77(5), 505-520 (2017).

3. Eshaghian S, Berenson JR. Multiple myeloma: improved outcomes with new therapeutic approaches. Curr. Opin. Support. Palliat. Care 6(3), 330-336 (2012).

4. Stewart AK, Rajkumar SV, Dimopoulos MA et al. Carfilzomib, lenalidomide, and dexamethasone for relapsed multiple myeloma. $N$. Engl. J. Med. 372(2), 142-152 (2015).

5. Moreau P, Masszi T, Grzasko N et al. Oral Ixazomib, lenalidomide, and dexamethasone for multiple myeloma. N. Engl. J. Med. 374(17), 1621-1634 (2016).

6. San Miguel J, Weisel K, Moreau P et al. Pomalidomide plus low-dose dexamethasone versus high-dose dexamethasone alone for patients with relapsed and refractory multiple myeloma (MM-003): a randomised, open-label, Phase III trial. Lancet Oncol. 14(11), 1055-1066 (2013).

7. Richardson PG, Siegel DS, Vij R et al. Pomalidomide alone or in combination with low-dose dexamethasone in relapsed and refractory multiple myeloma: a randomized Phase II study. Blood 123(12), 1826-1832 (2014).

8. Morgan GJ, Davies FE, Gregory WM et al. Effects of induction and maintenance plus long-term bisphosphonates on bone disease in patients with multiple myeloma: the Medical Research Council Myeloma IX Trial. Blood 119(23), 5374-5383 (2012).

9. Quach H, Ritchie D, Stewart AK et al. Mechanism of action of immunomodulatory drugs (IMiDS) in multiple myeloma. Leukemia 24(1), 22-32 (2010).

10. Niesvizky R, Martin TG, 3rd, Bensinger WI et al. Phase Ib dose-escalation study (PX-171-006) of carfilzomib, lenalidomide, and low-dose dexamethasone in relapsed or progressive multiple myeloma. Clin. Cancer Res. 19(8), 2248-2256 (2013).

11. Jakubowiak AJ, Dytfeld D, Griffith KA et al. A Phase I/II study of carfilzomib in combination with lenalidomide and low-dose dexamethasone as a frontline treatment for multiple myeloma. Blood 120(9), 1801-1809 (2012).

12. Richardson PG, Weller E, Lonial $S$ et al. Lenalidomide, bortezomib, and dexamethasone combination therapy in patients with newly diagnosed multiple myeloma. Blood 116(5), 679-686 (2010).

13. Hulin C, Hansen T, Heron L et al. Living with the burden of relapse in multiple myeloma from the patient and physician perspective. Leuk. Res. 59, 75-84 (2017).

14. Leleu X, Martin TG, Einsele H et al. Role of proteasome inhibitors in relapsed and/or refractory multiple myeloma. Clin. Lymphoma Myeloma Leuk. 19(1), 9-22 (2019).

15. Brenner H, Gondos A, Pulte D. Recent major improvement in long-term survival of younger patients with multiple myeloma. Blood 111(5), 2521-2526 (2008).

16. Mateos MV, San Miguel JF. Management of multiple myeloma in the newly diagnosed patient. Hematol. Am. Soc. Hematol. Educ. Program 2017(1), 498-507 (2017).

17. Dingli D, Ailawadhi S, Bergsagel PL et al. Therapy for relapsed multiple myeloma: guidelines from the mayo stratification for myeloma and risk-adapted therapy. Mayo Clin. Proc. 92(4), 578-598 (2017).

18. Siegel D, Martin T, Nooka A et al. Integrated safety profile of single-agent carfilzomib: experience from 526 patients enrolled in 4 Phase II clinical studies. Haematologica 98(11), 1753-1761 (2013).

19. Mushtaq A, Kapoor V, Latif A et al. Efficacy and toxicity profile of carfilzomib based regimens for treatment of multiple myeloma: a systematic review. Crit. Rev. Oncol. Hematol. 125, 1-11 (2018).

20. Moreau P, Mateos MV, Berenson JR et al. Once weekly versus twice weekly carfilzomib dosing in patients with relapsed and refractory multiple myeloma (A.R.R.O.W.): interim analysis results of a randomised, Phase III study. Lancet Oncol. 19(7), 953-964 (2018).

21. Dimopoulos MA, Goldschmidt H, Niesvizky R et al. Carfilzomib or bortezomib in relapsed or refractory multiple myeloma (ENDEAVOR): an interim overall survival analysis of an open-label, randomised, Phase III trial. Lancet Oncol. 18(10), 1327-1337 (2017).

22. Lonial S, Dimopoulos M, Palumbo A et al. Elotuzumab therapy for relapsed or refractory multiple myeloma. N. Engl. J. Med. 373(7), 621-631 (2015).

23. Deckert J, Wetzel MC, Bartle LM et al. SAR650984, a novel humanized CD38-targeting antibody, demonstrates potent antitumor activity in models of multiple myeloma and other CD38+ hematologic malignancies. Clin. Cancer Res. 20(17), 4574-4583 (2014).

-. A preclinical study reporting the mechanisms of action of isatuximab (ISA), highlighting the potent antitumor activity of this agent in cell lines and mouse models of multiple myeloma and other $\mathrm{CD}^{+} 8^{+}$hematologic malignancies.

24. Lonial S, Weiss BM, Usmani SZ et al. Daratumumab monotherapy in patients with treatment-refractory multiple myeloma (SIRIUS): an open-label, randomised, Phase II trial. Lancet 387(10027), 1551-1560 (2016). 
25. Lokhorst HM, Plesner T, Laubach JP et al. Targeting CD38 with daratumumab monotherapy in multiple myeloma. $N$. Engl. J. Med. 373(13), 1207-1219 (2015).

26. Richardson PG, Jagannath S, Moreau P et al. Elotuzumab in combination with lenalidomide and dexamethasone in patients with relapsed multiple myeloma: final Phase II results from the randomised, open-label, Phase Ib-2 dose-escalation study. Lancet Haematol. 2(12), e516-527 (2015).

27. Jelinek T, Hajek R. Monoclonal antibodies - a new era in the treatment of multiple myeloma. Blood Rev. 30(2), 101-110 (2016).

28. Krejcik J, Casneuf T, Nijhof IS et al. Daratumumab depletes CD $38^{+}$immune regulatory cells, promotes T-cell expansion, and skews T-cell repertoire in multiple myeloma. Blood 128(3), 384-394 (2016).

29. Chari A, Suvannasankha A, Fay JW et al. Daratumumab plus pomalidomide and dexamethasone in relapsed and/or refractory multiple myeloma. Blood 130(8), 974-981 (2017).

30. Mateos MV, Dimopoulos MA, Cavo M et al. Daratumumab plus bortezomib, melphalan, and prednisone for untreated myeloma. $N$. Engl. J. Med. 378(6), 518-528 (2018).

31. Dimopoulos MA, Dytfeld D, Grosicki S et al. Elotuzumab plus pomalidomide and dexamethasone for multiple myeloma. N. Engl. J. Med. 379(19), 1811-1822 (2018).

32. Feng $\mathrm{X}$, Zhang L, Acharya $\mathrm{C}$ et al. Targeting CD38 suppresses induction and function of $\mathrm{T}$ regulatory cells to mitigate immunosuppression in multiple myeloma. Clin. Cancer Res. 23(15), 4290-4300 (2017).

-• A preclinical study reporting the effect of ISA on immunosuppressive Tregs. ISA treatment may restore the immune function against multiple myeloma cells.

33. Moreno L, Zabaleta A, Alignani D et al. Critical analysis on the mechanism of action (MoA) of the anti-CD38 monoclonal antibody isatuximab in multiple myeloma (MM). Presented at: American Society of Hematology 58th Annual Meeting \& Exposition. Blood, CA, USA (2016).

34. Richardson PG, Attal M, Campana F et al. Isatuximab plus pomalidomide/dexamethasone versus pomalidomide/dexamethasone in relapsed/refractory multiple myeloma: ICARIA Phase III study design. Future Oncol. 14(11), 1035-1047 (2017).

35. Kuhn DJ, Chen Q, Voorhees PM et al. Potent activity of carfilzomib, a novel, irreversible inhibitor of the ubiquitin-proteasome pathway, against preclinical models of multiple myeloma. Blood 110(9), 3281-3290 (2007).

36. Dimopoulos MA, Bringhen S, Anttila P et al. Results from a Phase II study of isatuximab as a single agent and in combination with dexamethasone in patients with relapsed/refractory multiple myeloma. Presented at: American Society of Hematology 60th Annual Meeting CA, USA (2018).

37. Martin T, Strickland S, Glenn M et al. Phase I trial of isatuximab monotherapy in the treatment of refractory multiple myeloma. Blood Cancer J. 9(4), 41 (2019).

-• A Phase I study reporting the effect of ISA monotherapy. ISA monotherapy was active and tolerated in heavily pretreated patients with relapsed/refractory multiple myeloma.

38. Richter JR, Martin TG, Vij R et al. Updated data from a Phase II dose finding trial of single agent isatuximab (SAR650984, anti-CD38 $\mathrm{mAb}$ ) in relapsed/refractory multiple myeloma (RRMM). Presented at: American Society of Clinical Oncology 52nd Annual Meeting. IL, USA, 3-7 June 2016.

39. Martin T, Baz R, Benson DM et al. A Phase Ib study of isatuximab plus lenalidomide and dexamethasone for relapsed/refractory multiple myeloma. Blood 129(25), 3294-3303 (2017).

-. A Phase Ib study reporting the effect of ISA in combination with lenalidomide/dexamethasone. ISA in combination with lenalidomide/dexamethasone was tolerated and was active in heavily pretreated patients with relapsed/refractory multiple myeloma.

40. Richter JR, Martin TG, Vij R et al. Updated data from a Phase II dose finding trial of single-agent isatuximab (SAR650984, anti-CD38 $\mathrm{mAb}$ ) in relapsed/refractory multiple myeloma. In: American Society of Clinical Oncology 52nd Annual Meeting. IL, USA 3-7 June 2016.

-. A Phase II study reporting the effect of ISA monotherapy. ISA monotherapy was active and tolerated in heavily pretreated patients with relapsed/refractory multiple myeloma.

41. Attal M, Richardson PG, Rajkumar SV et al. Isatuximab plus pomalidomide and low-dose dexamethasone versus pomalidomide and low-dose dexamethasone in patients with relapsed and refractory multiple myeloma (ICARIA-MM): a randomised, multicentre, open-label, Phase 3 study. Lancet 37(10214), 2096-2107 (2019).

-• A Phase III study reporting the effect of ISA in combination with pomalidomide/dexamethasone. ISA in combination with pomalidomide/dexamethasone was tolerated and was active in heavily pretreated patients with relapsed/refractory multiple myeloma.

42. Chari A, Richter JR, Shah N et al. Phase I-b study of isatuximab + carfilzomib in relapsed and refractory multiple myeloma (RRMM). J. Clin. Oncol. 36(Suppl. 15), 8014-8014 (2018).

43. Kumar S, Paiva B, Anderson KC et al. International Myeloma Working Group consensus criteria for response and minimal residual disease assessment in multiple myeloma. Lancet. Oncol. 17(8), e328-e346 (2016). 
44. Sun Z, Zheng F, Wu S, Liu Y, Guo H, Liu Y. Triplet versus doublet combination regimens for the treatment of relapsed or refractory multiple myeloma: a meta-analysis of Phase III randomized controlled trials. Crit. Rev. Oncol. Hematol. 113, 249-255 (2017).

45. Palumbo A, Chanan-Khan A, Weisel K et al. Daratumumab, bortezomib, and dexamethasone for multiple myeloma. N. Engl. J. Med. 375(8), 754-766 (2016).

46. Dimopoulos MA, Oriol A, Nahi H et al. Daratumumab, lenalidomide, and dexamethasone for multiple myeloma. N. Engl. J. Med.375(14), 1319-1331 (2016).

47. Spencer A, Lentzsch S, Weisel $\mathrm{K}$ et al. Daratumumab plus bortezomib and dexamethasone versus bortezomib and dexamethasone in relapsed or refractory multiple myeloma: updated analysis of CASTOR. Haematologica 103(12), 2079-2087 (2018).

48. Bahlis N, Dimopoulos MA, White DJ et al. Three-year follow up of the Phase III pollux study of daratumumab plus lenalidomide and dexamethasone (D-Rd) versus lenalidomide and dexamethasone (Rd) alone in relapsed or refractory multiple myeloma (RRMM). Blood 132(Suppl. 1), 1996-1996 (2018).

49. Chari A, Martinez-Lopez J, Mateos MV et al. Daratumumab plus carfilzomib and dexamethasone in patients with relapsed or refractory multiple myeloma. Blood 134(5), 421-431 (2019). 